\title{
Subcutaneous emphysema and pneumothorax after using jet injector during Microscopic laryngeal polypectomy : a case report and literature review
}

\author{
Gi-hyeon Jang ${ }^{1}$, Ja-Sook Kim ${ }^{2}$ and Ju-hwan Lee ${ }^{1,3}$ \\ ${ }^{1}$ Department of Anesthesiology and Pain Medicine, Wonkwang University School of Medicine, Wonkwang \\ University Hospital, Iksan, Korea \\ ${ }^{2}$ Department of Nursing, Donggang University, Gwangju, Korea \\ ${ }^{3}$ Jesaeng-Euise Clinical Anatomy Center, Wonkwang University School of Medicine, Iksan, Korea
}

\begin{abstract}
Background/Objectives: A 64 year old male patient was scheduled to receive microscopic laryngeal polypectomy to remove a mass located anterior vocal cord $(0.3 \times 0.5 \mathrm{~cm})$. Methods/Statistical analysis: A 5.5 french wired anode tube was tried to intubate but the airway anatomy was not suitable to intubation (short chin and protruded maxilla ) and large size epiglottis so intubation was failed. Jet injector was performed through vocal cord posterior wall and injector was intubated but worried tracheal trauma. Findings: The surgery was started and polypectomy was successfully completed, but subcutaneous emphysema, pneumothorax occurred. we tried to prevent life threatening events such as upper airway obstruction, tension pneumothorax, pneumopericardium, mediastinitis might be possible immediately tracheostomy was performed. Improvements/Applications: after that we find the reason of subcutaneous emphysema and we find mucosal perforation of below vocal cord area located in upper airway.
\end{abstract}

\section{Index Terms}

Intubation; Pneumo-mediastinum; Subcutaneous enphysema; Jet injector; polypectomy

Corresponding author : Ju-hwan Lee

fesjil@naver.com

- Manuscript received January 10, 2020.

- Revised February 10, 2020 ; Accepted March 2, 2020.

- Date of publication March 30, 2020.

(C) The Academic Society of Convergence Science Inc.

2619-8363 $\odot 2020$ IJBSA. Personal use is permitted, but republication/redistribution requires IJBSA permission. 


\section{INTRODUCTION}

There is a possibility that an experienced anesthesiologist may cause some damage to the airway even if he or she intubates in patients with difficult airway. However, complications such as pneumomediastinum, subcutaneous emphysema can make Mediastinitis, tension pneumothorax, pericardial abscess, caused by improper oxygen supply, brain death, cardiac arrest, cardiac arrest, etc. must be avoided[1]. Jet injector (VBM Manujet III) may be useful for maintaining respiration during surgery for patients with airway problems[2,3]. Most of reported mediastial injury occurred after intubate difficult airway patient $[4,5,6]$ and vomiting and coughing during recovery [7] tonsillectomy[8], thyroidectomy, using guard oxygen conduit[9], facial mask [10], aspirator [11], using rigid bronchoscopy [12], but there was no report in the case of hard jet injector. The author reports Subcutaneous emphysema and pneumothorax after using jet injector during Microscopic laryngeal polypectomy

In Korea, there have not been reports of Subcutaneous emphysema and pneumothorax after using jet injector during Microscopic laryngeal polypectomy. Herein, we report the case with a literature review.

\section{CASE}

A 64 years old male patient visit hospital difficulty in breathing from five months ago, he was diagnosed with vocal cord polyp. The polyp was $0.3 \times 0.5 \mathrm{~cm}$ in size and the facial anatomy of patient was guessed difficult intubation and the scheduled surgery was to conduct polypectomy performed under general anesthesia (Fig.1). The operation was expected to be simple within 10 minutes and decided to try using the Anode Tube with a small internal diameter. He had no surgical skills in the past, no family history and no past findings, and was a patient of Class 2 in ASA classification. Ondansetron $8 \mathrm{mg}$ oral administration was given 1 hour before the onset of anesthesia, and ECG, non-invasive automatic blood pressure cuff and pulse oxygen meter were attached after arrival in the operating room. Preoperative vital signs showed blood pressure $130 / 81 \mathrm{mmHg}$, heart rate 75 times/minutes, oxygen saturation $97 \%$. In order to induce anesthesia, $100 \mathrm{mg}$ of propofol and $100 \mathrm{mg}$ of succinylcholine chloride were injected and $0.1 / \mathrm{g} / \mathrm{kg} / \mathrm{min}$ of Refientanil was continuously infused during operation. After confirming that the patient's muscle relaxation was sufficient, an intubation was attempted. The difficulty of intubation is level 3 on Cormack and Lehane classification, and only the tip behind the epiglottis is visible. $5.0 \mathrm{~mm}$ inner diameter Anode Tube was used to intubate, but it failed to pass through the vocal cord because of airway anatomy. Insertion using a surgical metallic jet injector with an internal diameter of $2.0 \mathrm{~mm}$ was attempted, and the tube was passed vocal cord and check breathing sounds from both lungs were of the same sound (Fig.2). The anesthesia was maintained by using Jet injector (VBM Manujet III) to maintain total intravenous anesthesia by injecting $0.2 \mathrm{~L} / \mathrm{min}$ of oxygen, 20 $\mathrm{mg} / \mathrm{hr}$ of propofol, and $0.1 \mu \mathrm{g} / \mathrm{kg} / \mathrm{min}$ of Remifentanil. Vital signs and blood pressure were normal. For surgery the ridged laryngoscope was inserted and the surgical metallic jet injector was placed to the right side. polypectomy under the microscopy was conducted immediately, a sudden pneumomediastinum and subcutaneous emphysema occurred, and the jet injector was immediately removed. Ventilation using mask was attempted, but proper ventilation was not achieved due to subcutaneous air throughout the face, and oxygen saturation measured using the patient's pulse oximetry decreased by $80 \%$. An emergency tracheostomy was performed and ventilation was carried out using Tracheostomy Tube (the Blue Line, SIMS Portex Ltd, UK). Oxygen saturation recovered to $85 \%$ but was no longer elevated, but both lung breathing sounds were not heard in the stethoscope due to subcutaneous emphysema, and pneumothorax in the right lung were immediately detected during emergency chest radiography (Fig 3), and chest tube inserted immediately to right chest. after pneumothorax were recovered, breathing sounds were heard on the both side of lung, and pulse oxygen saturation was recovered to $95 \%$. About five minutes after stopping the injection of anesthetic drugs, spontaneous respiration recovered, and the patient was moved to the intensive care unit to maintain $96 \%$ pulse oxygen saturation with a catheter $(2-5 \mathrm{~L})$ at the entrance of the tracheotomy. The next day, the Pneumomediastinum and pneumothorax were almost lost, and the vital signs of normal levels and pulse oxygen saturation were maintained at $99 \%$ and discharged after a week of polypectomy surgery.

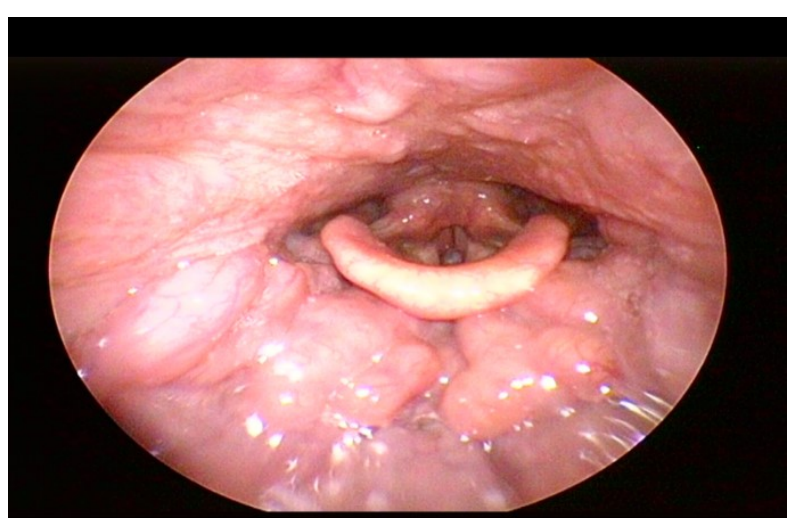

Fig. 1. laryngoscopic images shows vocal cord polyp( 0.3 


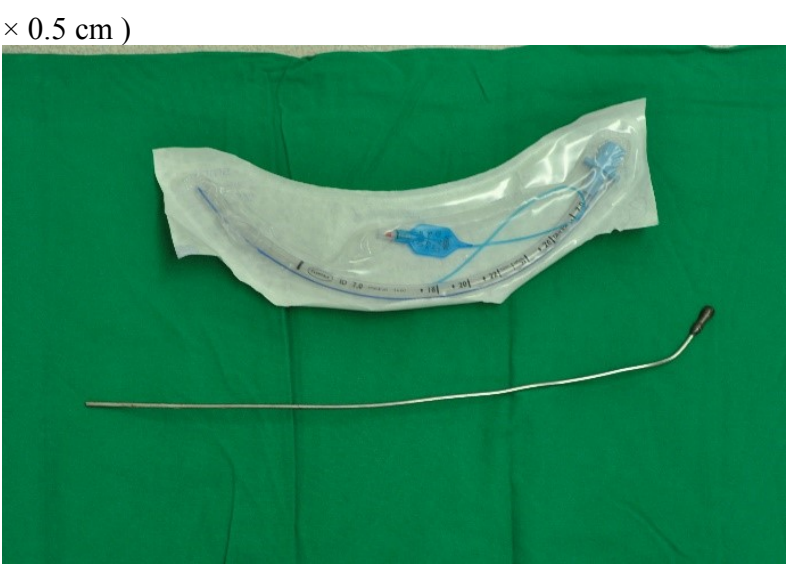

Fig. 2. Metal endotracheal tube $(2.0 \mathrm{~mm}$ internal diameter $)$ lower. polyvinylchloride endotracheal tube $(7.0 \mathrm{~mm}$ internal diameter) upper.

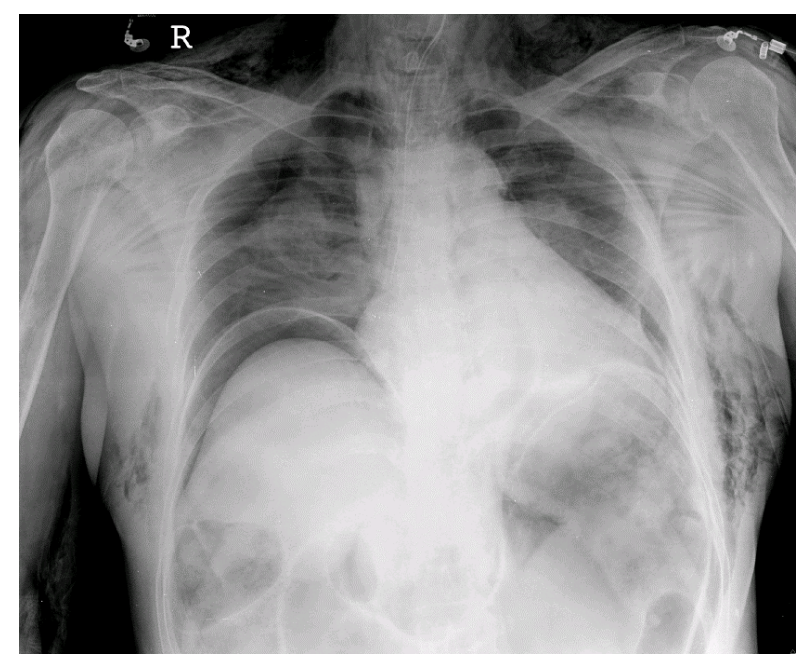

Fig. 3. chest X-ray shows free air below diaphragm on both lung and pneumothrox on Rt. lung

\section{DisCUSSION}

The cervical fascia of the neck is divided into a shallow layer and a deep layer, and the deep layer forms the boundary of the potential space of the neck. The visceral layer, one of the deep layers, wraps the organs, esophagus, and major neck vessels, and goes down into the longitudinal cavity to wrap the bronchial tubes, bronchial arteries, lymphatic tubes, gimp joints and heart lungs.

One of the mechanisms for the occurrence of an Subcutaneous emphysema is the rupture of air bubbles around the vessel due to an increase in intraalveolar pressure, which leads to pleural and mediastinal spaces. The other mechanism may be caused by traumatic disruption of the thoracic wall or pericardial membrane.13) In the latter case, when air enters the pericardial space of the head and neck through incision, laceration, and exudation site, the pericardial membrane is dissected and the pericardium enters the retropharyngeal space and the mediastinal space make pneumothorax, pneumopericardium, and pneumo-peritoneum. Through subcutaneous tissue and loose connective tissue pathways, air flows down to the abdominal wall and scrotum and up to the head from neck, face and head.

In this patient's case, the causes of pneumomediastinum and subcutaneous emphysema can be divided into upper airways and bronchial damage, and rupture of small airways or pulmonary edema. In this case, the patient was a 64-year-old male patient who was rather difficult to manipulate the airway during anesthesia induction and was easy to iatrogenic damage if not paid attention. The anesthesiologist who conducted the intubation while attempting intubation several times may made damage with immature or rough intubation methods. And there is also the possibility of damaging the trachea branch or bronchial mucous membrane during intubation of intraoperative metal tubes. Also, the most likely cause of bronchial mucous membrane damage is excessive angle manipulation in the process of moving the tube to the right to avoid rigid laryngoscope while positioning for surgery. Finally, the possibility of air inflow through the polypectomy site cannot be ruled out as pressure is applied to the polypectomy area during microscopic surgery. Jet injector is more likely to cause airway damage because they have a larger outer diameter than the polyvinylchloride tubes of hard and identical internal diameter. Immediately after anesthesia induction, ventilation was difficult due to the high maximum air intake pressure caused by stenosis, which may have caused pressure damage (barotrauma). And during anesthesia, the end tidal carbon dioxide level is higher than endotracheal tube pressure, so it has no choice but to maintain a higher suction gas flow than necessary. Therefore, the internal pressure of the lung can be increased, resulting in auto-PEEP, which can cause pressure damage, such as incipient airway or pulmonary rupture. After blind suction with Yankauer type-sucker to remove airway secretions before anesthesia was performed without difficulty during surgery for strapedectomy, a small rupture of the pharyngeal cavity that required surgical treatment and a life-threatening upper phase also experienced closure [11]. $91 \mathrm{kPa}$ or $68 \mathrm{kPa}$ were said to be suitable for suction, which is aspiration of rectal mucosa. Patients in this example used aspirators during surgery with a maximum sound pressure of $70 \mathrm{kPa}$. Thus, it may be possible to cause the suction catheter itself to move or to cause the mucous membrane tissue to fail due to a strong suction force 
$[14,15]$

Airway damage can be easily caused, especially if neck and head movements are excessive when positioning for surgery, or if excessive angular fragments of a tube in a metal organ. The rigid bronchoscope reported dysplasia and evasive species that occurred after the removal of child care tissue in the left main organ site, which is similar to the author's experience that the airway was manipulated with a hard tube [12].

In this case, there are reports of similar complications without any obvious difficulty in intubation. Pneumomediastinum and submucosal empysema that occurred after vomiting and coughing after the release of the vessel, and found a small lesion on the back wall of the organ on the third day after surgery. Many doctors argued that tracheal intubation was the most frequent and most likely cause of Pneumomediastinum and submucosal empysema. And it is recommended to perform checking the artificial lesions with an endoscope immediately, but even during the surgery, the causative lesions were not identified by the bronchial endoscope due to tumors in the bronchial tubes. In the recovery room, the subclavian model caused by a mucous membrane rupture and the complete upper respiratory tract also experienced closure. The gas supplied to the airways under constant pressure enters a small break in the mucous membrane and is isolated through the tissue surface, causing subcutaneous and mucous substrate[9].

In this case, excessive intra-tube pressure, internal wall damage caused by metal intra-tube, and damage caused by surgery could be the cause and could be fatal in the event of complications. During surgery of various airway stenosis patients, maintaining the patient's breathing is difficult for anesthesiologists, and sensitive manipulation is needed to prevent complications from occurring. Surgery requires the use of non-pressed rigid metallic tubes, and the Anode Tube is a non-pressed, curved tube that can be useful for this instance. However, the Anode tube, which is currently on the market, is difficult to use because it is made for infants and is not long in length for small inner diameter tubes. Therefore, it is thought that a long $(25 \mathrm{~cm}-28 \mathrm{~cm})$ internal diameter (3.0-5.0 $\mathrm{mm})$ tube will be needed for these operations. Also, proper pressure maintenance is required when ventilating positive pressure using jet injector to maintain oxygen saturation.

\section{CONCLUSION}

In conclusion, in order to maintain adequate ventilation in emergency situations such as those experienced in this case, it is necessary to expose and disinfect the neck chest area during surgery and to prepare an emergency organ intubation.

\section{REFERENCES}

[1] Benumof JL (1996). Airway management: principles and practice. St. Louis: Mosby-year Book, Inc. 886

[2] Ayechu, MG, Tajadura, JJ, N. Ramirez, ET, Berruezo, MA (2007). Jet ventilation (Manujet) in fibroptic bronchoscopy treatment of postoperative atelectasis. Revista Espanola de Aensth. 54(6), 386-387

[3] Benumof, JL. \& Scheller, Mark S. M.D (1989). The Importance of Transtracheal Jet Ventilation in the Management of the Difficult Airway Anesthesiology 71, 769-778

[4] Moller GM, Berge EJFM, Stassen CM. (1994). Tracheocele: a rare cause of difficult endotracheal intubation and subsequent pneumomediastinum. Eur Respir J. 7, 1376-7

[5] Hyun soo Moon at. el. (1993). A case of esophageal perforation during endotracheal intubation in ankylosing cervical rheumatoid patient. Korean J. of Aanesth. 26, 1289-93

[6] Young ho jang at. El. (1994). Subcutaneous Emphysema Caused by Tracheal Intubation. Korean J. of Aanesth. 27, 1205-9

[7] Obenhaus T. (1996). Postoperative pneumomediastimum and emphysema after anesthesia with endotracheal intubation. Anesthesiol Intensivemed Notfallmed Schmerter. 31, 318-320

[8] Smelt GJC. (1984). Subcutaneous emphysema: Pathological and anesthetic, but not surgical. $J$. Laryngol Otol. 98, 647-654

[9] Larsen KD. (1988). submucosal emphysema with airway obstruction from nasal wxygen cannula. Anesth Analg. 67, 586-7

[10] Grime PD, Malins TJ. (1991): Hazard warning. A case of postoperative pulmonary barotrauma. Brit $J$ oral Max Sug 29, 183-4

[11] Bellamy MC, Berridge JC, Hussain SSM. (1993). Surgical emphysema and upper airway obstruction complicating recovery from anesthesia. Brit J Anesth. $71,592-3$

[12] Redleaf MI. (1995). Pneumomediastinum after rigid bronchoscopy. Ann Oto Rhinol Laryn. 104, 955-6

[13] Reiche FO. (1995). Intraoperative life threatening emphysema associated with endotracheal intubation and air insufflation devices. J Oral Maxil Surg. 53, 1103-7

[14] Brandborg LL, Rubin GE, Quinton WE. (1995). Multipurpose instrument for suction biopsy of esophagus, stomach, small bowel, and colon. Gastroenterology. 37, 1-16

[15] Noblett HR. (1996). A rectal suction biopsy tube for use in the diagnosis of Hirschprung's disease. 4, 4105 